\section{Reply to Letter of Shukla and Milsom}

\section{TO THE EDITORS:}

Drs. Shukla and Milsom raise important issues about the management of advanced cancer and the potential value of coding systems that record the extent and type of metastatic disease. They recommend basing this coding on the tumornode-metastasis (TNM) system by using a more robust "rTNM" (recurrent) and introducing an "mTNM" (metastatic).

Certainly, advances in surgical and nonsurgical care along with improved supportive care provide viable and positive options for patients who previously had very limited choice. Existing prognostic schemas may not be sufficiently discriminatory to define prognosis accurately for all these patients. Shukla and Milsom illustrate this point through hypothetical cases of advanced colorectal cancer that would share the same TNM classification but receive different treatment and might have different prognosis.

TNM includes a system to report "recurrent stage" using the same T, N, and M codes as for initial cancer stage. Of course, the rTNM classifies all those with any distant metastases as M1 or stage IV. Prognosis or at least length of survival may vary widely within the M1 classification. Current TNM for some diseases include M1 subgroups based on the type and extent of metastatic disease. Examples include colorectal cancer based on the number of sites of metastases (M1a, single metastatic site; M1b, multiple metastatic sites) and melanoma based on the sites of metastases and serum lactate dehydrogenase (LDH) level (M1A; M1B; M1C). Shukla presents cases that intuitively suggest that these M1 classifications are not sufficient.

The American Joint Committee on Cancer (AJCC) welcomes suggestions on ways to enhance the robustness of TNM, including subclassification of metastases and recurrent cancer. The AJCC taskforces specifically examined these issues and used best available data in their $7^{\text {th }}$ edition deliberations. Unfortunately, while differences in prognosis between individual cases within a classification may seem apparent, outcome data are required to establish a codification system. Such data may be hard to find. Current national registry datasets such as the Surveillance, Epidemiology, and End Results Program (SEER) and the National Cancer Data Base (NCDB) do not have the degree of detail on extent of metastases to identify and distinguish between such cases. Therefore, to allow revisions to the staging system, any available datasets at large centers need to be tapped or new datasets need to be developed.

For the 7th edition AJCC Cancer Staging Manual, there were a number of circumstances where existing registry data were insufficient to support needed revisions. In some of these diseases, international collaborative groups established data collection programs to define needed revisions. These included melanoma, lung cancer, esophageal cancer, and gastric cancer. Each of these dedicated groups identified the need, developed the collaboration, and did the work to collect data or aggregate existing data. This is the kind of effort needed to enhance the classification of advanced colorectal cancer.

The AJCC cannot itself finance and conduct all the work needed for these efforts. We encourage Shukla and Milsom or others to take on this task in advanced colorectal cancer. Moving forward, the AJCC will increase its support through advice and counsel, and potentially limited financial support, to those conducting this work. It will assist in convening experts, support workshops, and provide a clearing house for access to results to hasten the development of new prognostic and predictive models for improving cancer treatment and outcome. Doing this to help define treatment and outcome for those with advanced disease is indeed a worthy mission.

Stephen B. Edge, MD, and Carolyn Compton, MD, PhD American Joint Committee on Cancer, Chicago, IL e-mail: stephen.edge@ roswellpark.org

Published Online: 16 October 2010

(C) Society of Surgical Oncology 2010 